H. Fujimoto

Nagoya Math. J.

V01. 33 (1968), 85-106

\title{
ON THE HOLOMORPHIC AUTOMORPHISM GROUPS OF COMPLEX SPACES
}

\author{
HIROTAKA FUJIMOTO
}

\section{\$1. Introduction.}

For a complex space $X$ we consider the group Aut $(X)$ of all automorphisms of $X$, where an automorphism means a holomorphic automorphism, i.e. an injective holomorphic mapping of $X$ onto $X$ itself with the holomorphic inverse. In 1935, H. Cartan showed that Aut $(X)$ has a structure of a real Lie group if $X$ is a bounded domain in $C^{N}([7])$ and, in 1946, S. Bochner and D. Montgomery got the analogous result for a compact complex manifold $X$ ([2] and [3]). Afterwards, the latter was generalized by R.C. Gunning ([11]) and H. Kerner ([16]), and the former by W. Kaup ([14]), to complex spaces. The purpose of this paper is to generalize these results to the case of complex spaces with weaker conditions. For brevity, we restrict ourselves to the study of $\sigma$-compact irreducible complex spaces only.

The main results are the followings.

Theorem A. Let $X$ be a $\sigma$-compact irreducible complex space such that $X-K$ is BK-complete for a compact set $K$ in $X$ (see Definition 5.1 of \$5). Then, endowed with the compact open topology, Aut $(X)$ is locally compact (Theorem 5. 3).

According to Bochner-Montgomery [2], it then follows that Aut $(X)$ has a structure of a Lie group. For the examples of such spaces, see Example 5.4 of $\S 5$.

Theorem B. If a $\sigma$-compact irreducible complex space $X$ is *-strongly pseudoconcave (see Definition 8.1 of $\S 8$ ), then Aut $(X)$ is locally compact and so a Lie group (Theorem 8. 3).

For example, if $Y$ is a compact, connected $N$-dimensional complex manifold and $M$ is an analytic set of embedding dimension $\leqq N-2$, the space $X:=Y-M$ is $*$-strongly pseudo-concave.

Received January 24, 1968. 
We study first normal families of holomorphic mappings with images in $K$-complete spaces (\$2). And we give some fundamental properties of sequences of nowhere degenerate holomorphic mappings for later uses, generalizing the results of $\mathrm{C}$. Carathéodory ([5]) to the case of complex spaces (\$3).

Next we show that, for an arbitrary $\sigma$-compact $X$, Aut $(X)$, with the compact open topology, is a topological group which is complete with respect to the canonical uniform structure ${ }^{1)}$ (Theorem 4. 1). Each $\varphi \in$ Aut $(X)$ induces canonically the automorphism of $X_{r}$, where $X_{r}$ denotes the manifold of all regularities of $X$. We shall prove that $\operatorname{Aut}(X)$ can be topologically identified with a closed subgroup of Aut $\left(X_{r}\right)$ if $X$ is $K$-complete (Theorem 4. 3).

In $\S 5$, we give the precise formulation of Theorem $\mathrm{A}$ and study some related problem. Theorem $A$ is completely proved in the end of $\$ 6$. We give a convergence theorem of holomorphic mappings of a *-strongly pseudo-concave open set in $\$ 7$ and, using this, prove Theorem B in $\$ 8$.

\section{\$2. Normal families of holomorphic mappings.}

Let $X$ and $Y$ be (reduced) complex spaces. We consider the space $\mathrm{Hol}(X, Y)$ of all holomorphic mappings of $X$ into $Y$ endowed with the compact open topology, namely, the weakest topology such that for each compact $K \subset X$ and open $U \subset Y$ the set $W(K, U):=\{\varphi \in \operatorname{Hol}(X, Y) ; \varphi(K) \subset$ $U\}$ is open. For complex spaces $X$ and $Y$ with countable topology, Hol $(X, Y)$ has also a countable topology. In this case, the topology of $Y$ is given by a metric $\rho$. The topology of $\operatorname{Hol}(X, Y)$ is equal to that of compact convergence, namely, the topology such that $\lim _{n} \varphi_{n}=\varphi\left(\varphi_{n}, \varphi \in \mathrm{Hol}\right.$ $(X, Y))$ is defined as $\lim _{n} \sup _{x \in K} \rho\left(\varphi_{n}(x), \varphi(x)\right)=0$ for any compact $K \subset X$. Sometimes, we consider the space $\operatorname{Hol}(X, Y)$ with the topology of compact convergence referred to an arbitrarily pre-assigned metric $\rho$ on $Y$.

As is well known, if a family $\mathscr{F}$ of holomorphic functions on a complex space is uniformly bounded on each compact $K$ in $X, \mathscr{F}$ is normal, namely, any sequence in $\mathscr{F}$ has a convergent subsequence. We generalize this to the case of holomorphic mappings.

Theorem 2. 1. Let $X$ and $Y$ be $\sigma$-compact complex spaces and assume that

1) This means the uniform structure defined by the family of the sets $\tilde{\mathfrak{U}}=\{(\phi, \psi) \in \operatorname{Aut}(X)$ $\times \operatorname{Aut}(X) ; \varphi \psi^{-1} \in \mathfrak{U}$ and $\left.\varphi^{-1} \psi \in \mathfrak{U}\right\}$ for neighborhoods $\mathfrak{U}$ of the identity in Aut $(X)$. 
$Y$ is $K$-complete. A family $\Phi$ in $\mathrm{Hol}(X, Y)$ is relatively compact in $\mathrm{Hol}(X, Y)$ if and only if, for any compact $K$ in $Y, \Phi(K):=\{\varphi(x) ; x \in K, \varphi \in \Phi\}$ is relatively compact in $Y$.

For the proof, we recall the following well-known theorem (e.g. Bourbaki $[4], \S 2)$.

Theorem. For a locally compact space $X$ and a metric space $Y$ let $C(X, Y)$ be the space of all continuous mappings of $X$ into $Y$ endowed with the topology of compact convergence. A family $\Phi$ in $C(X, Y)$ is relatively compact in $C(X, Y)$ if and only if for any $x \in X$ (i) $\Phi(x)$ is relatively compact and (ii) $\Phi$ is equicontinuous at $x$.

Proof of theorem 2. 1. The canonical mapping $F(\varphi, x):=\varphi(x)$ of $\mathrm{Hol}(X, Y)$ $\times X$ into $Y$ is continuous in $\varphi$ and $x$ simultaneously. For any relatively compact $\Phi \subset \mathrm{Hol}(X, Y)$ and compact $K \subset X, \Phi(K)$ is relatively compact in $Y$ as the $F$-image of a relatively compact set $\Phi \times K$.

As is easily shown, $\operatorname{Hol}(X, Y)$ is closed in $C(X, Y)$. To see the converse assertion, it suffices to show that $\Phi$ is relatively compact in $C(X, Y)$. Now, we assume that $\Phi$ is not equicontinuous at some $x_{0} \in X$. Let $\left\{U_{n}\right\}$ be a countable base of relatively compact, connected neighborhoods of $x_{0}$. By the assumption, we can take a real $\delta>0$ such that for each $n$ there exist a point $x_{n} \in U_{n}$ and $\varphi_{n} \in \Phi$ with $\rho\left(\varphi_{n}\left(x_{n}\right), \varphi_{n}\left(x_{0}\right)\right) \geqq \delta$. Since any $\varphi_{n}\left(x_{0}\right)$ is contained in a relatively compact $\Phi\left(U_{1}\right)$, we may assume $\left\{\varphi_{n}\left(x_{0}\right)\right\}$ converges to a point $y_{0} \in Y$. By the definition of $K$-completeness, there exist holomorphic functions $f_{1}, \cdots, f_{k}$ on $Y$ such that for some neighborhood $V$ of $y_{0} \bar{V} \cap\left\{f_{1}=\cdots=f_{k}=0\right\}=\left\{y_{0}\right\}$, where we assume

$$
\bar{V} \subset\left\{y ; \rho\left(y_{0}, y\right)<\frac{\delta}{2}\right\}
$$

for later uses. Then each sequence $\left\{f_{i} \varphi_{n} ; n=1,2, \cdots\right\}(1 \leqq i \leqq k)$ is uniformly bounded on $U_{1}$ because

$$
\sup \left\{\left|f_{i}\left(\varphi_{n}(x)\right)\right| ; x \in U_{1}\right\} \leqq \sup \left\{\left|f_{i}(y)\right| ; y \in \Phi\left(\bar{U}_{1}\right)\right\}<+\infty .
$$

Hence it has a subsequence which converges on $U_{1}$. Without loss of generality, we may assume that each $\left\{f_{i} \varphi_{n}\right\}$ itself converges to a holomorphic function $g_{i}$ on $U_{1}$. Since $\lim _{n} \varphi_{n}\left(x_{0}\right)=y_{0}$, we have

$$
g_{i}\left(x_{0}\right)=\lim _{n}\left(f_{i} \varphi_{n}\right)\left(x_{0}\right)=\lim _{n} f_{i}\left(\varphi_{n}\left(x_{0}\right)\right)=f_{i}\left(y_{0}\right)=0
$$


and there exists some $n_{0}$ such that $\varphi_{n}\left(x_{0}\right) \in V$ for any $n \geqq n_{0}$. Then it follows

$$
\rho\left(\varphi_{n}\left(x_{n}\right), y_{0}\right) \geqq \rho\left(\varphi_{n}\left(x_{n}\right), \varphi_{n}\left(x_{0}\right)\right)-\rho\left(\varphi_{n}\left(x_{0}\right), y_{0}\right) \geqq \delta-\frac{\delta}{2}=\frac{\delta}{2}
$$

for any $n \geqq n_{0}$, which shows $\varphi_{n}\left(x_{n}\right) \notin V$. Accordingly, $\varphi_{n}\left(U_{n}\right) \cap V \neq \phi$ and, at the same time, $\varphi_{n}\left(U_{n}\right)-V \neq \phi$ for each $n \geqq n_{0}$. Thus the connected $\varphi_{n}\left(U_{n}\right) \quad\left(n \geqq n_{0}\right)$ has to intersect the boundary $\partial V$ of $V$. We take a sequence $\left\{x_{n}^{\prime}\right\}$ such that $x_{n}^{\prime} \in U_{n}$ and $\varphi_{n}\left(x_{n}^{\prime}\right) \in \partial V$. Obviously, $\lim _{n} x_{n}^{\prime}=x_{0}$ and so $\lim _{n}\left(f_{i} \varphi_{n}\right)\left(x_{n}^{\prime}\right)=g_{i}\left(x_{0}\right)$. On the other hand, by the compactness of $\partial V$, $\left\{\varphi_{n}\left(x_{n}^{\prime}\right)\right\}$ has a subsequence $\varphi_{n_{k}}\left(x_{n_{k}}^{\prime}\right)$ converging to a point $y^{\prime} \in \partial V$. Then, we have

$$
f_{i}\left(y^{\prime}\right)=\lim _{k} f_{i}\left(\varphi_{n_{k}}\left(x_{n_{k}}^{\prime}\right)\right)=\lim _{k}\left(f_{i} \varphi_{n_{k}}\right)\left(x_{n_{k}}^{\prime}\right)=g_{i}\left(x_{0}\right)=0
$$

for any $i(1 \leqq i \leqq k)$. This contradicts the assumption $\bar{V} \cap\left\{f_{i}=0\right\}=\left\{y_{0}\right\}$. In conclusion, $\Phi$ is equicontinuous at any $x \in X$. By the above theorem, $\Phi$ is relatively compact in $C(X, Y)$ because the condition (i) is obviously satisfied. The proof is completed.

§3. Some properties of nowhere degenerate holomorphic mappings.

For brevity, we restrict ourselves to the study of holomorphic mappings between $\sigma$-compact complex spaces of the same dimension. In the following sections, complex spaces are always assumed to be $\sigma$-compact and of puredimension $N$.

By definition, a holomorphic mapping $\varphi$ of a complex space $X$ into another $Y$ is non-degenerate at $x \in X$ if and only if $\operatorname{dim}_{x} \varphi^{-1} \varphi(x)=0$. A non-degenerate mapping means a mapping which is non-degenerate at some $x \in X$ and a nowhere degenerate mapping means a mapping which is nondegenerate at any $x \in X$.

For complex spaces $X$ and $Y$ we denote their normalizations by $X^{*}$ and $Y^{*}$ respectively. With each nowhere degenerate holomorphic mapping 0 we can associate the uniquely determined holomorphic mapping $\mu^{*}(\varphi)$ of $X^{*}$ into $Y^{*}$ with $\varphi \cdot \mu_{1}=\mu_{2} \cdot \mu^{*}(\varphi)$, where $\mu_{1}$ and $\mu_{2}$ are the projection mappings.

Lemma 3. 1. Let $\varphi$ and $\varphi_{n}(n \geqq 1)$ be nowhere degenerate holomorphic map- 
pings of $X$ into $Y$. The sequence $\left\{\varphi_{n}\right\}$ converges to $\varphi$ in $\operatorname{Hol}(X, Y)$ if and only if $\left\{\mu^{*}\left(\varphi_{n}\right)\right\}$ converges to $\mu^{*}(\varphi)$ in $\operatorname{Hol}\left(X^{*}, Y^{*}\right)$.

Proof. For brevity, we put $\varphi_{n}^{*}:=\mu^{*}\left(\varphi_{n}\right)$ and $\varphi^{*}:=\mu^{*}(\varphi)$. Assume that $\lim _{n} \varphi_{n}^{*}=\varphi^{*}$ and take an arbitrary compact $K \subset X$ and an open $U \subset Y$ with $\varphi \in W(K, U)$. Then, $\varphi^{*}\left(\mu_{1}^{-1}(K)\right) \subset \mu_{2}^{-1}(U)$. Since $\mu_{1}^{-1}(K)$ is compact, it follows $\varphi_{u}^{*}\left(\mu_{1}^{-1}(K)\right) \subset \mu_{2}^{-1}(U)$ for almost all $n$. For these $n$, we have

$$
\varphi_{n}(K)=\varphi_{n}\left(\mu_{1}\left(\mu_{1}^{-1}(K)\right)\right)=\mu_{2} \varphi_{u}^{*}\left(\mu_{1}^{-1}(K)\right) \subset \mu_{2}\left(\mu_{2}^{-1}(U)\right)=U .
$$

This shows $\lim _{n} \varphi_{n}=\varphi$.

Conversely, let $\lim _{n} \varphi_{n}=\varphi$. For any $x \in X$ we choose relatively compact neighborhoods $U$ of $x$ and $V$ of $\varphi(x)$ such that $\varphi(\bar{U}) \subset V \Subset W$ with some $K$-complete open subset $W$ of $Y$. By the assumption, $\varphi_{n}(U) \subset V$ and so $\varphi_{n}^{*}\left(\mu_{1}^{-1}(U)\right) \subset \mu_{2}^{-1}(V)$ for almost all $n$. Since $\mu_{2}^{-1}(V) \Subset \mu_{2}^{-1}(W)$ and $\mu_{2}^{-1}(W)$ is $K$-complete, $\left\{\varphi_{1}^{*}\right\}$ has a subsequence converging on $\mu_{1}^{-1}(U)$ by Theorem 2. 1. By the usual diagonal argument, we can choose a subsequence of $\left\{\varphi_{n_{b}}^{*}\right\}$ which converges to $\psi$ in $\mathrm{Hol}\left(X^{*}, Y^{*}\right)$. The limit $\psi$ satisfies $\mu_{2} \psi$ $=\varphi \cdot \mu_{1}=\mu_{2} \varphi^{*}$ because $\mu_{2} \varphi_{n}^{*}=\varphi_{n} \mu_{1}$. Since $\mu_{2}$ is injective on a everywhere dense open subset of $Y^{*}$, we conclude $\psi=\varphi^{*}$, which is determined independently of any choice of the convergent subsequence of $\left\{\varphi_{n}^{*}\right\}$. Thus, $\left\{\varphi_{n}^{*}\right\}$ itself converges to $\varphi^{*}$ in $\operatorname{Hol}\left(X^{*}, Y^{*}\right)$.

Lemma 3. 2. Let $\left\{\varphi_{n}\right\}$ be a sequence of nowhere degenerate holomorphic mappings of $X$ into $Y$ which converges to a mapping $\varphi$ in $\operatorname{Hol}(X, Y)$. If $\varphi$ is nowhere degenerate on $X$, for any open sets $G_{1}, G_{2}$ and $G_{3}$ with $G_{1} \Subset G_{2} \Subset G_{3}$, it holds

$$
\varphi_{m}\left(\bar{G}_{1}\right) \subset \varphi\left(G_{2}\right) \subset \varphi\left(\bar{G}_{2}\right) \subset \varphi_{n}\left(G_{3}\right)
$$

for almost all $m$ and $n$.

Proof. At first, we assume that $X$ and $Y$ are normal. By the assumption, any $x \in \bar{G}_{2}$ has a relatively compact neighborhood $U$ with $\varphi^{-1}(\varphi(x)) \cap \bar{U}$ $=\{x\}$. Since $\varphi(\partial U) \nsubseteq \varphi(x)$ and compact, we can take a connected neighborhood $W$ of $\varphi(x)$ with $\varphi(\partial U) \cap \bar{W}=\phi$. We put $V=\varphi^{-1}(W) \cap \bar{U}$. As is easily seen, the restriction $\varphi \mid V$ of $\varphi$ to $V$ is a proper nowhere degenerate mapping into $W$. Then, it holds $\varphi(V)=W$ by the normality of $Y$. While, since $\lim _{n} \varphi_{n}=\varphi, \varphi_{n}(\partial U) \cap \bar{W}=\phi$ and $\varphi_{n}(x) \in W$ for almost all $n$. For these $n$, putting $U_{n}^{\prime}:=\varphi_{n}^{-1}(W) \cap U$, we see $\varphi(V)=W=\varphi_{n}\left(U_{n}^{\prime}\right) \subset \varphi_{n}\left(G_{3}\right)$. Thus, 
with each $x \in \bar{G}_{2}$ we can associate a neighborhood $V_{x}$ of $x$ such that $\varphi\left(V_{x}\right)$ $\subset \varphi_{n}\left(G_{3}\right)$ for any $n \geqq n(x)$ with a suitable integer $n(x)$. By the compactness of $\bar{G}_{2}$, we can select finitely many $V_{x_{i}}$ such that $\bar{G}_{2} \subset \cup_{i=1}^{r} V_{x_{i}}$. Obviously, $\varphi_{n}\left(G_{3}\right) \supset \varphi\left(\bar{G}_{2}\right)$ for any $n \geqq \max \left\{n_{x_{i}} ; 1 \leqq i \leqq r\right\}$.

Now, we suppose $\varphi_{n}\left(\bar{G}_{1}\right) \not \subset \varphi\left(G_{2}\right)$ for infinitely many $n$. There exist a subsequence $\left\{\varphi_{n_{k}}\right\}$ of $\left\{\varphi_{n}\right\}$ and points $x_{k} \in \bar{G}_{1}$ such that $\varphi_{n_{k}}\left(x_{k}\right) \notin \varphi\left(G_{2}\right)$. Since $\bar{G}_{1}$ is compact, we may assume that $\left\{x_{k}\right\}$ converges to a point $x_{0}$ in $\bar{G}_{1}$. While, $\varphi\left(G_{2}\right)$ is open in $Y$ by the result of R. Remmert [19], p. 358. We see $\varphi\left(x_{0}\right)=\lim _{k} \varphi_{n_{k}}\left(x_{k}\right) \notin \varphi\left(G_{2}\right)$. This is a contradiction. In conclusion, $\varphi_{n}\left(\bar{G}_{1}\right) \subset \varphi\left(G_{2}\right)$ for almost all $n$. This completes the proof of Lemma 3.2 for normal complex spaces $X$ and $Y$.

To prove Lemma 3. 2 for arbitrary $X$ and $Y$, we consider the normalizations $X^{*}$ and $Y^{*}$ of $X$ and $Y$ with projection mappings $\mu_{1}$ and $\mu_{2}$ respectively and use the same notations as in the proof of Lemma 3.1. The mappings $\varphi^{*}, \varphi_{n}^{*} \in \operatorname{Hol}\left(X^{*}, Y^{*}\right)$ and open sets $G_{i}^{*}:=\mu_{1}^{-1}\left(G_{i}\right)(i=1,2,3)$ in $X^{*}$ satisfy all assumptions in Lemma 3.2 in view of Lemma 3.1. By the above proof, we have

$$
\varphi_{m}^{*}\left(\bar{G}_{1}^{*}\right) \subset \varphi^{*}\left(G_{2}^{*}\right) \subset \varphi^{*}\left(\bar{G}_{2}^{*}\right) \subset \varphi_{n}^{*}\left(G_{3}^{*}\right)
$$

for almost all $m$ and $n$. Immediately, we conclude

$$
\varphi_{m}\left(\bar{G}_{1}\right) \subset \varphi\left(G_{2}\right) \subset \varphi\left(\bar{G}_{2}\right) \subset \varphi_{n}\left(G_{3}\right)
$$

because $\mu_{2} \varphi_{n}^{*}\left(G_{i}^{*}\right)=\varphi_{n}\left(G_{\imath}\right)$ and $\mu_{2} \varphi_{n}^{*}\left(\bar{G}_{i}^{*}\right)=\varphi_{n}\left(\bar{G}_{i}\right)$ etc. . Lemma 3. 2 is completely proved.

Proposition 3. 3. Let $\left\{\varphi_{n}\right\}$ be a sequence of biholomorphic mappings of a complex space $X$ onto open subsets (depending on each $\varphi_{n}$ ) of a fixed complex space $Y$. If $\left\{\varphi_{n}\right\}$ converges to a nowhere degenerate $\varphi$ in $\operatorname{Hol}(X, Y)$, then it holds the followings:

(i) The image $\varphi(X)$ is open and $\varphi$ is a biholomorphic mapping of $X$ onto $\varphi(X)$.

(ii) The sequence $\left\{\varphi_{n}^{-1}\right\}$ of the inverses of $\varphi_{n}$ converges to $\varphi^{-1}$ on $\varphi(X)$.

Remark. According to Lemma 3.2, any open set $D \Subset \varphi(X)$ satisfies that, for almost all $n, \varphi_{n}(X) \supset D$ and so $\varphi_{n}^{-1} \mid D$ is well-defined. In Proposition 3. $3, \lim _{n} \varphi_{n}^{-1}=\varphi^{-1}$ on $\varphi(X)$ means that for any $D \Subset \varphi(X) \lim _{n} \varphi_{n}^{-1} \mid D$ $=\varphi^{-1} \mid D$. 
Proof of Proposition 3. 3. To see the injectivity of $\varphi$, take arbitrary points $x_{1}$ and $x_{2}\left(x_{1} \neq x_{2}\right)$ in $X$ and neighborhoods $U_{1}$ of $x_{1}$ and $U_{2}$ of $x_{2}$ with $U_{1} \cap U_{2}=\phi$. By Lemma 3. $2 \varphi\left(x_{i}\right) \in \varphi_{n}\left(U_{i}\right)(i=1,2)$ for almost all $n$. So, we can take points $y_{i} \in U_{i}$ such that $\varphi\left(x_{i}\right)=\varphi_{n}\left(y_{i}\right)$ for a suitable $n$. By the assumption, $y_{1} \neq y_{2}$ concludes $\varphi_{n}\left(y_{1}\right) \neq \varphi_{n}\left(y_{2}\right)$ and so $\varphi\left(x_{1}\right) \neq \varphi\left(x_{2}\right)$. This shows that $\varphi$ is injective.

Now, take an arbitrary open set $D$ in $X$. For any $x \in D$ we assign a relatively compact neighborhood $U$ of $x$ with $\bar{U} \subset D$. Applying Lemma 3. 2 again, we see $\varphi(x) \in \varphi_{n}(U) \subset \varphi(D)$ for almost all $n$. This shows that $\varphi(D)$ is open. Therefore, $\varphi$ is open and, in particular, $\varphi(X)$ is open. Moreover, this shows also the continuity of the mapping $\varphi^{-1}$ of $\varphi(X)$ onto $X$. To see the holomorphy of $\varphi^{-1}$, it suffices to prove the assertion (ii) because the limit of $\varphi_{n}^{-1}$ is holomorphic.

Let $K$ be a compact subset of $\varphi(X)$ and $U$ be an open set in $X$ such that $\varphi^{-1} \in W(K, U)$, i.e. $K \subset \varphi(U)$. Since $\varphi^{-1}(K)$ is compact, by Lemma 3. 2 we have $K=\varphi\left(\varphi^{-1}(K)\right) \subset \varphi_{n}(U)$, i.e. $\varphi_{n}^{-1} \in W(K, U)$ for almost all $n$. This concludes $\lim _{n} \varphi_{n}^{-1}=\varphi^{-1}$ on $\varphi(X)$.

\section{\$4. The automorphism group of a complex space.}

Let $X$ be a complex space. By Aut $(X)$, we denote the group of all automorphisms of $X$. With the topology induced from $\operatorname{Hol}(X, X), \operatorname{Aut}(X)$ is a Hausdorff space with a countable topology because $X$ is assumed to be $\sigma$-compact. The composite mapping $H(\varphi, \psi)=\varphi \cdot \psi$ is a continuous mapping of the product space Aut $(X) \times \operatorname{Aut}(X)$ into Aut $(X)$. Moreover, in Proposition 3. 3 considering the case that $X=Y$ and $\varphi_{n}$ and $\varphi$ are surjective, we see $\operatorname{Aut}(X)$ is a topological group.

In addition, we can prove

Theorem 4. $1^{2)}$. For any $\sigma$-compact purely dimensional complex space $X$ Aut $(X)$ is a topological group which is complete with respect to the canonical uniform structure.

Proof. Take a Cauchy sequence $\left\{\varphi_{n}\right\}$ in Aut $(X)$. We shall prove first $\left\{\varphi_{n}\right\}$ has a convergent subsequence as a sequence in $\mathrm{Hol}(X, X)$. To

2) Added in Proof. This can be proved by the only property that $X$ is locally compact and locally connected, in view of the following paper: R. Arens, Topologies for homeomorphism groups, Amer. J. Math., 68 (1946), 593-610. 
this end, for any $x \in X$ we take neighborhoods $U$ and $V$ of $x$ such that $\bar{U} \subset V \Subset W$ with some $K$-complete open subset $W$ of $X$. The set $W(\bar{U}, V)$ is a neighborhood of the identity automorphism $\varepsilon$ in Aut $(X)$. By the definition of a Cauchy sequence we can find a positive integer $n_{0}$ such that $\varphi_{m}^{-1} \varphi_{n}(\bar{U}) \subset V$ for any $m, n \geqq n_{0}$. Putting $m=n_{0}$, we have $\varphi_{n}(\bar{U}) \subset \varphi_{n_{0}}(V)$ $\Subset \varphi_{n_{0}}(W)$, where $\varphi_{n_{0}}(W)$ is $K$-complete because $\varphi_{n_{0}}$ is an automorphism of $X$. By Theorem 2.1 we can select a subsequence of $\left\{\varphi_{n} \mid U\right\}$ which converges in $\mathrm{Hol}(U, X)$. Covering $X$ by at most countably many such $U^{\prime}$, we can choose a subsequence of $\left\{\varphi_{n}\right\}$ which converges in $\operatorname{Hol}(X, X)$ by the usual diagonal argument.

On the other hand, $\left\{\varphi_{n}^{-1}\right\}$ is also a Cauchy sequence. Applying the same argument as above to $\left\{\varphi_{n}^{-1}\right\}$, we get a subsequence $\left\{\varphi_{n_{k}}\right\}$ of $\left\{\varphi_{n}\right\}$ such that $\lim _{k} \varphi_{n_{k}}=\varphi$ and $\lim _{k} \varphi_{n_{k}}^{-1}=\psi$ for some $\varphi, \phi \in \operatorname{Hol}(X, X)$. Obviously, $\varphi \cdot \psi=\psi \cdot \varphi=\varepsilon$ on $X$. Therefore, we conclude $\lim _{k} \varphi_{n_{k}}=\varphi \in \operatorname{Aut}(X)$. In general, if a Cauchy sequence has a subsequence with the limit $\varphi$, it converges to the same limit $\varphi$. The given $\left\{\varphi_{n}\right\}$ itself converges to $\varphi$ in Aut $(X)$. This completes the proof.

Let $X$ be a complex space and $X^{*}$ be its normalization with projection mapping $\mu$. Each $\varphi \in \operatorname{Aut}(X)$ gives an automorphism $\mu^{*}(\varphi) \in \operatorname{Aut}\left(X^{*}\right)$ with $\varphi \cdot \mu=\mu \cdot \mu^{*}(\varphi)$. Thus we obtain a mapping $\mu^{*}: \operatorname{Aut}(X) \rightarrow \operatorname{Aut}\left(X^{*}\right)$, which is obviously injective.

Proposition 4. 2. By the mapping $\mu^{*}$, Aut $(X)$ is topologically isomorphic with a closed subgroup of Aut $\left(X^{*}\right)$.

Proof. By Lemma 3. 1, the group homomorphism $\mu^{*}$ is uniformly bicontinuous of $\operatorname{Aut}(X)$ onto a topological subgroup $\mu^{*}(\operatorname{Aut}(X))$ of $\operatorname{Aut}\left(X^{*}\right)$ with respect to the canonical uniform structure. In view of Theorem 4. 1, $\mu^{*}(\operatorname{Aut}(X))$ is complete and therefore closed in $\operatorname{Aut}\left(X^{*}\right)$.

An automorphism $\varphi$ of $X$ maps a regular point to a regular point of $X$. We denote the set of all regularities of $X$ by $X_{r}$. Each $\varphi \in \operatorname{Aut}(X)$ induces the automorphism of $X_{r}$. We get the injective group homomorphism $\rho: \operatorname{Aut}(X) \rightarrow \operatorname{Aut}\left(X_{r}\right)$.

Theorem 4. 3. If $X$ is K-complete, Aut $(X)$ is identified with a closed subgroup of Aut $\left(X_{r}\right)$ by the mapping $\rho$. 
For the proof, we need

Lemma 4. 4. Take a sequence $\left\{\varphi_{n}\right\}$ of automorphisms of a $K$-complete complex space $X$. For a thin analytic subset $M$ of $X$, if $\left\{\varphi_{n}\right\}$ converges to a nowhere degenerate $\varphi$ in $\operatorname{Hol}(X-M, X),\left\{\varphi_{n}\right\}$ converges on the total $X$ to a mapping $\psi \in \operatorname{Hol}(X, X)$.

Proof. Firstly, we note that under the assumption of $K$-completeness of $X$ any $x \in X$ has a fundamental system of neighborhoods each of which is $X$-convex. By definition, we can take finitely many holomorphic functions $f_{1}, \cdots, f_{k}$ on $X$ such that $\left\{f_{1}=\cdots=f_{k}=0\right\} \cap \bar{V}=\{x\}$ for a sufficiently small relatively compact neighborhood $V$ of $x$. For any $\delta<\inf _{y \in \partial V} \max$ $\left\{\left|f_{i}(y)\right| ; 1 \leqq i \leqq k\right\}$, the set

$$
U:=\left\{\left|f_{1}\right|<\delta, \cdots,\left|f_{k}\right|<\delta\right\} \cap V
$$

is $X$-convex. Because, any compact $K \subset U$ satisfies

$$
\begin{gathered}
\hat{K} \cap U:=\left\{x \in U ;|f(x)| \leqq \sup _{y \in K}|f(y)| \text { for any holomorphic } f \text { on } X\right\} \\
\subset\left\{x ;\left|f_{i}(x)\right| \leqq \delta^{\prime}, 1 \leqq i \leqq k\right\} \cap U
\end{gathered}
$$

with $\delta^{\prime}:=\sup \left\{\left|f_{i}(y)\right| ; y \in K, 1 \leqq i \leqq k\right\}<\delta$, which is compact.

Now, for any $x \in M$ we take a relatively compact $X$-convex neighborhood $U$ of $x$. We shall prove that there exist a compact set $K$ and a connected compact set $L$ such that $K \cap M=\phi, K \subset L \subset \hat{K} \subset U$ and $L$ contains $x$ as an interior point (c.f. H. Grauert and R. Remmert [10], Hilfssatz 4, p. 292). As is well known, there exists a neighborhood $V(\subset U)$ such that a suitable proper nowhere degenerate holomorphic mapping $\pi=\left(\pi_{1}, \cdots, \pi_{N}\right)$ maps $V$ onto a polydisc $P:=\left\{\left|z_{1}\right|<1, \cdots,\left|z_{N}\right|<1\right\}$ in $C^{N}(N=\operatorname{dim} X)$ and satisfies $\bar{V} \cap \pi^{-1} \pi(x)=\{x\}$. Furthermore, we can choose these $V, \pi$ and $P$ so that they satisfy

$$
\pi(M) \subset\left\{\left|z_{1}\right|<s,\left|z_{2}\right|<1, \cdots,\left|z_{N}\right|<1\right\}
$$

for some $s<1$. We put

$$
\begin{aligned}
K & =\pi^{-1}\left(\left\{s \leqq\left|z_{1}\right| \leqq r,\left|z_{2}\right| \leqq r, \cdots,\left|z_{N}\right| \leqq r\right\}\right), \\
L & =\pi^{-1}\left(\left\{\left|z_{1}\right| \leqq r,\left|z_{2}\right| \leqq r, \cdots,\left|z_{N}\right| \leqq r\right\}\right),
\end{aligned}
$$

where $s<r<1$. Obviously, $L$ is connected compact and contains $x$ as an 
interior point. Moreover, it is easy to prove every holomorphic function on a neighborhood of $L$ attains its maximum value at a point in $K$. This shows $L \subset \hat{K}$ and hence these $K$ and $L$ satisfy the desired conditions.

Take an open set $G$ such that $G \Subset U, G \cap M=\phi$ and $K \subset G$. Then, since $\varphi$ is nowhere degenerate on $X-M$, according to Lemma 3. 2 we have

$$
\varphi_{n}(K) \subset \varphi(G) \subset \varphi_{m}(U-M) \subset \varphi_{m}(U)
$$

for any $m, n \geqq n_{0}$ with a suitable $n_{0}$. Since $U$ is $X$-convex and $\varphi_{n_{0}}$ is an automorphism of $X, \varphi_{n_{0}}(U)$ is also $X$-convex. Therefore, $\widehat{\varphi_{n}(K)} \cap \varphi_{n_{0}}(U)$ is compact and $\varphi_{n}(L)\left(\subset \varphi_{n}(\hat{K})\right)$ is a compact connected subset of $\widehat{\varphi_{n}(K)}$. For any $n \geqq n_{0}, \varphi_{n}(K) \subset \varphi_{n_{0}}(U)$ implies $\varphi_{n}(L) \subset \widehat{\varphi_{n}(K)} \cap \varphi_{n_{0}}(U)$. Because, if not, the connected $\varphi_{n}(L)$ is represented as the disjoint union of non-empty compact sets $\varphi_{n}(L) \cap \varphi_{n_{0}}(U)$ and $\varphi_{n}(L)-\varphi_{n_{0}}(U)$. In conclusion, there exists a neighborhood $W(\subset L)$ of $x$ such that $\varphi_{n}(W) \subset \varphi_{n_{0}}(U) \Subset X$ for almost all $n$. In virtue of Theorem 2.1, we can find a subsequence $\left\{\varphi_{n_{k}}\right\}$ which converges on $W$ to $\psi_{W} \in \operatorname{Hol}(W, X)$ with $\psi_{W}=\varphi$ on $W-M$. Since $M$ is thin, any convergent subsequence of $\left\{\varphi_{n} \mid W\right\}$ has the same limit $\psi_{W}$ on $W$ and so $\left\{\varphi_{n}\right\}$ itself converges to $\psi_{W}$ on $W$. Moreover, by the same reason, $\psi_{W}=\psi_{W^{\prime}}$ on $W \cap W^{\prime}$ for any choice of the above $x$ and $W$ if $W \cap W^{\prime} \neq \phi$. Putting $\psi=\psi_{W}$ on each $W$, we define $\psi \in \operatorname{Hol}(X, X)$. The sequence $\left\{\varphi_{n}\right\}$ converges to $\varphi$ in $\operatorname{Hol}(X, X)$.

Proof of Theorem 4.3. Obviously, $\rho$ is continuous. Our aim is to show that for a sequence $\left\{\varphi_{n}\right\}$ in $\operatorname{Aut}(X)$, if $\lim _{n} \varphi_{n}=\varphi$ in $\operatorname{Aut}\left(X_{r}\right)$, it converges also on $X$ to an automorphism of $X$. Since $\varphi$ is injective, we may apply Lemma 4.4 to the thin analytic set of all singularities of $X$. There exists a holomorphic mapping $\psi \in \operatorname{Hol}(X, X)$ with $\lim _{n} \varphi_{n}=\psi$ on $X$. While, in virtue of Proposition 3. 3, we see $\lim _{n} \varphi_{n}^{-1} \mid X_{r}=\varphi^{-1}$ on $X_{r}$. Using Lemma 4. 4 again, we obtain $\psi^{\prime} \in \operatorname{Hol}(X, X)$ such that $\lim _{n} \varphi_{n}^{-1}=\psi^{\prime}$ on $X$. Obviously, $\psi \cdot \psi^{\prime}=\psi^{\prime} \cdot \psi=\varepsilon$ on $X$ and so $\psi \in$ Aut $(X)$. This completes the proof.

\section{§5. The automorphism groups of complex spaces of some bounded} types.

For convenience' sake, we give the following 
Definition 5. 1. We shall call a complex space $X$ to be $B K$-complete if for any $x \in X$ there exist finitely many bounded holomorphic functions $f_{1}, \cdots, f_{k}$ on $X(1 \leqq i \leqq k)$ such that $\left\{f_{1}=\cdots=f_{k}=0\right\}$ contains $x$ isolatedly.

Propositron 5.2. It is a necessary and sufficient condition for the BKcompleteness of a complex space $X$ that $X$ can be considered as a bounded Riemann domain, namely, there exists a nowhere degenerate holomorphic mapping $\pi$ of $X$ into a bounded set in $C^{N}(N=\operatorname{dim} X)$.

Proof. The sufficiency is evident. To see the necessity, we have only to show that for some $l(\geqq N)$ there exist $l$ bounded holomorphic functions $f_{1}, \cdots, f_{l}$ on $X$ which give a nowhere degenerate mapping $f=\left(f_{1}, \cdots, f_{l}\right)$ of $X$ into $C^{l}$ (see H. Grauert [9], Satz 11, p. 252 and its proof). We consider the set $B(X)$ of all bounded holomorphic functions on $X$. Endowed with the uniform topology, $B(X)$ is a Banach space. By mathematical induction on $k$, we shall show that for any $k$-dimensional analytic set $M$ in $X$ there exists a mapping $f: X \rightarrow C^{l}\left(f \in B(X)^{l}\right)$ which is nowhere degenerate on $M$, where $B(X)^{l}$ is the $l$-fold direct sum of $B(X)$. If $\operatorname{dim} M=0$, the proof is evident. Assume this for the case of $\operatorname{dim} M \leqq k-1$. Let $M$ be of dimension k. For each irreducible component $M_{i}$ of $M$, the set

$$
\mathscr{B}_{i}:=\left\{f=\left(f_{1}, \cdots, f_{k}\right) \in B(X)^{k} ; f \text { is non-degenerate on } M_{i}\right\}
$$

is obviously non-empty and open in $B(X)^{k}$ (c.f. [9], Hilfssatz 3, p. 245). For any $f \in B(X)^{k}$, putting $h_{i}=f_{i}+c_{i} g_{i}$ with $g=\left(g_{i}\right) \in \mathscr{B}_{i}$, we get a mapping $h=\left(h_{1}, \cdots, h_{k}\right) \in \mathscr{B}_{i}$ if sufficiently small $c_{i}$ are suitably chosen. This shows $\mathscr{B}_{i}$ is dense in $B(X)^{k}$. While, $B(X)^{k}$ is a Baire space. There is a mapping $f: X \rightarrow C^{k}$ such that $f \in \cap_{i} \mathscr{B}_{i}$ which is non-degenerate on any $M_{i}$. Then, the set $E:=\left\{x \in M\right.$; $\left.\operatorname{dim}_{x} f^{-1} f(x)>0\right\}$ is a thin analytic subset of $M$ and so $\operatorname{dim} E \leqq k-1$. By the induction hypothesis, a suitable $g=\left(g_{1}, \cdots, g_{l}\right) \in B(X)^{l}$ is nowhere degenerate on $E$. Then, the mapping $h=\left(f_{1}, \cdots, f_{k}, g_{1}, \cdots, g_{l}\right): X \rightarrow C^{k+l}$ is nowhere degenerate on $M$. Our purpose is accomplished. In particular, if $k=N=\operatorname{dim} X$, we may consider $X$ itself as the above $M$. This completes the proof of Proposition 5. 2.

Now, we give the following theorem, which is a generalization of the H. Cartan's theorem ([7], Theorem 13, p. 50). 
Theorem 5. 3. Let $X$ be an irreducible complex space such that $X-K$ is $B K$-complete for some compact $K$ in $X$. Then the automorphism group $\operatorname{Aut}(X)$ is locally compact and so a Lie group.

For an irreducible complex space $X$, Aut $(X)$ is an effective analytic transformation group of the connected complex manifold $X_{r}$. The last assertion in Theorem 5. 3 is due to the following well-known theorem.

THEOREM. Let $G$ be an effective transformation group of class $C^{1}$ which acts on a connected differentiable manifold of class $C^{1}$. If $G$ is locally compact, $G$ is a Lie group (Bochner [2], Kuranishi [17] and Montgomery-Zippin [18]).

Before the accomplishment of the proof of Theorem 5. 3, we give some examples of complex spaces satisfying the condition in Theorem 5. 3 and study some related problem.

ExAmple 5. 4. (i) A bounded Riemann domain satisfies obviously the condition in Theorem 5. 3. For the case of a bounded Riemann domain without singularities, Theorem 5. 3 was proved by H. Cartan in [6] and [7]. Recently, W. Kaup proved this for an arbitrary $B K$-complete space $X$ in [14].

(ii) Let $Y$ be a compact complex space and $L$ be a compact set in $Y$ such that $Y-L$ is irreducible. If $L$ is included in some $K$-complete open subset $U$ of $Y$, the space $X:=Y-L$ satisfies also the condition in Theorem 5. 3. Indeed, if we put $K=X-V$ for another open $V$ with $L \subset V \Subset U$, $X-K=V-L$ is $B K$-complete. In the case that $L=\phi$, namely, $X$ is compact, Theorem 5. 3 was firstly proved by Bochner-Montgomery [2] for complex manifolds and afterwards generalized by R.C. Gunning [11] and H. Kerner [16] to arbitrary compact complex spaces.

Related to Example 5. 4 (ii), we have

Proposition 5. 5. Let $Y$ be a normal compact complex space of dimension $\geqq 2$ and $L$ be a compact set in $Y$. If $L$ has a fundamental system of neighborhoods $\left\{U_{n}\right\}$ such that one of them is Stein and each $U_{n}-L$ is connected, then for the space $X:=Y-L \operatorname{Aut}(X)$ is nothing but the closed subgroup of $\operatorname{Aut}(Y)$ consisting of all $\varphi \in \operatorname{Aut}(Y)$ with $\varphi(L)=L$.

Proof. We put $G=\{\varphi \in \operatorname{Aut}(Y) ; \varphi(L)=L\}$. The canonical restriction 
mapping $\rho: G \rightarrow \operatorname{Aut}(X)$ is obviously injective continuous homomorphism. Both $G$ and $\operatorname{Aut}(X)$ are complete topological groups with countable topology. If $\rho$ is surjective, $\rho$ is a topological isomorphism by the open mapping theorem (e.g. see T. Husain [12], p. 99). To see the surjectivity of $\rho$, we shall show that each $\varphi \in \operatorname{Aut}(X)$ can be continued to $\psi \in \operatorname{Aut}(Y)$.

Take a Stein neighborhood $U$ of $L$. For a given $\varphi \in \operatorname{Aut}(X)$, we can choose easily an open set $U^{\prime}$ in $Y$ such that $L \subset U^{\prime} \Subset U, \varphi\left(U^{\prime}-L\right) \subset U-L$, $\varphi^{-1}\left(U^{\prime}-L\right) \subset U-L$ and $U^{\prime}-L$ is connected by the assumption, The holomorphic mapping $\varphi \mid U^{\prime}-L$ has the image in a Stein space $U$. According to H. Kerner [15], Satz 2, p. 46, $\varphi$ is continuable to $U^{\prime}$ (c.f. [13] and [8]). Thus we obtain a mapping $\psi \in \operatorname{Hol}(Y, Y)$ with $\psi \mid U^{\prime}-L=\varphi$. By the same argument, there exists $\psi^{\prime} \in \operatorname{Hol}(Y, Y)$ with $\phi^{\prime} \mid Y-L=\varphi^{-1}$, which satisfies $\phi \psi^{\prime}=\psi^{\prime} \cdot \psi=\varepsilon$ on $Y$. This shows $\psi \in \operatorname{Aut}(Y)$. Thus, Proposition 5.5 is proved.

\section{\$6. The proof of the generalized H. Cartan's theorem.}

The purpose of this section is to prove Theorem 5.3 in the previous section. We shall show first

Proposition 6. 1. Let $X$ be an irreducible complex space such that $X-K$ is $B K$-complete for a compact $K$ in $X$. If a sequence $\left\{\varphi_{n}\right\}$ in $\operatorname{Aut}(X)$ converges to a nowhere degenerate holomorphic mapping $\varphi$ on an non-empty open set $D$ including $K$ such that $K \subset \varphi(D)$ and $\varphi(K) \subset D$, then $\left\{\varphi_{n}\right\}$ converges to a mapping $\tilde{\varphi}$ in $\operatorname{Hol}(X, X)$.

For the proof, we consider the set

$$
G=\left\{x \in X ;\left\{\varphi_{n}(x)\right\} \text { has an accumulation point in } X\right\} .
$$

Obviously, $G$ includes $D$.

Lemma 6. 2. Under the assumption in Proposition 6. 1, if for a sequence $\left\{x_{n}\right\}$ in $X \lim _{n} x_{n}=x_{0}$ and $\left\{\varphi_{n}\left(x_{n}\right)\right\}$ has an accumulation point in $X$, then a suitable subsequence of $\left\{\varphi_{n}\right\}$ converges on a neighborhood of $x_{0}$.

Proof. If $x_{0} \in D$, the proof is trivial. Let $x_{0} \notin D$. Without loss of generality, we may assume $\left\{\varphi_{n}\left(x_{n}\right)\right\}$ itself converges to a point $y_{0} \in X$. Then, we see $y_{0} \notin K$. Indeed, if $y_{0} \in K$, we have $x_{0}=\lim _{n} \varphi_{n}^{-1} \varphi_{n}\left(x_{n}\right)=\varphi^{-1}\left(y_{0}\right) \in D$ because $\lim _{n} \varphi_{n}^{-1}=\varphi^{-1}$ on $\varphi(D)$ ( $\supset K$ ) (c.f. Proposition 3. 3).

By Proposition 5.2, there exists a nowhere degenerate holomorphic 
mapping $\pi$ of $U:=X-K$ onto $\pi(U) \Subset C^{N}(N=\operatorname{dim} X)$. Taking an open $D^{\prime}$ such that $K \subset D^{\prime} \Subset D$, we see $K \subset \varphi_{n}\left(D^{\prime}\right)$ and $\varphi_{n}(K) \subset D^{\prime}$ for almost all $n$ by the assumption and Lemma 3.2. For these $n$, the mappings $\psi_{n}:=\pi \varphi_{n}$ and $\chi_{n}:=\pi \varphi_{n}^{-1}$ are well-defined on $U^{\prime}:=X-\bar{D}^{\prime}\left(U^{\prime} \supset X-D\right)$. Since $\psi_{n}\left(U^{\prime}\right)$ and $\chi_{n}\left(U^{\prime}\right)$ are included in $\pi(U) \Subset C^{N}$, after a suitable replacement of the indices, we may assume that both $\left\{\psi_{n}\right\}$ and $\left\{\chi_{n}\right\}$ converge to $\psi$ and $\chi$ on $U^{\prime}$ respectively in virtue of Theorem 2. 1. Now, we take arbitrarily small neighborhoods $W_{1}$ of $y_{0}$ and $W_{2}$ of $z_{0}:=\pi\left(y_{0}\right)$ such that $\pi \mid W_{1}: W_{1} \rightarrow W_{2}$ is proper, where we assume $W_{1} \Subset W$ with some $K$-complete open subset $W$ of $X$ for later uses. Moreover, we take a connected relatively compact neighborhood $V$ of $x_{0}$ such that $\psi(V) \subset W_{2}$. Then $\psi_{n}(V)=\pi \varphi_{n}(V) \subset W_{2}$, namely, $\varphi_{n}(V) \subset \pi^{-1}\left(W_{2}\right)$ for almost all $n$. We know that $W_{1}$ is open and closed in $\pi^{-1}\left(W_{2}\right)$. While, since $\lim _{n} \varphi_{n}\left(x_{n}\right)=y_{0}, \varphi_{n}(V) \cap W_{1} \neq \phi$ for almost all $n$. From these fact, we conclude $\varphi_{n}(V) \subset W_{1}$ for almost all $n$. Apply Theorem 2. 1 to the mappings $\varphi_{n} \mid V$. A suitable subsequence of $\left\{\varphi_{n}\right\}$ converges on $V$.

Lemma 6. 3. The set $G$ is open and there exists a subsequence of $\left\{\varphi_{n}\right\}$ which converges on $G$ to an injective mapping in $\operatorname{Hol}(G, X)$.

Proof. Each $x_{0} \in G$ satisfies the condition in Lemma 6. 2 for a sequence $\left\{x_{n}:=x_{0}\right\}$. So, $G$ is obviously open. While, the existence of a convergent subsequence of $\left\{\varphi_{n}\right\}$ is easily shown by the usual diagonal argument. To see the injectivity of the limit $\tilde{\varphi}$, we use the mapping $\chi=\lim _{n} \pi \varphi_{n}^{-1}$. By definition, $\pi=\chi_{n} \varphi_{n}$ on $X-D$ and so $\pi=\chi \tilde{\varphi}$ on $(X-D) \cap G$. The mapping $\tilde{\varphi}$ is nowhere degenerate on $D$ by the assumption and on $(X-D) \cap G$ because $\pi$ is nowhere degenerate. Therefore, $\tilde{\varphi}$ is nowhere degenerate and hence injective by Proposition 3.3 on the total $G$.

Lemma 6. 4. The set $G$ coincides with the total $X$.

Proof. The given sequence $\left\{\varphi_{n}\right\}$ may be assumed to have the properties as in the proof of Lemma 6.2 and converge to an injective holomorphic mapping $\tilde{\varphi}$ of $G$ into $X$ in view of Lemma 6. 3. Suppose that $G$ is a proper subset of $X$. We can choose a point $x_{0} \notin G$ which can be joined with a point in $G$ by a continuous curve $\gamma(t)(0 \leqq t \leqq 1)$ such that $\gamma(0)=x_{0}$ and $\gamma(t) \in G$ for any $t>0$. Indeed, joining a point $x_{1} \notin G$ with some $x_{2} \in G$ by a continuous curve $\gamma(t)\left(\gamma(0)=x_{1}, \gamma(1)=x_{2}\right)$, we take a point 
$x_{0}=\gamma\left(t_{0}\right)$ for $t_{0}:=\inf \{\tau ; \gamma(t) \in G$ for any $t>\tau\}$. After a suitable change of the parameter of the curve, the point has the desired property for $\gamma(t)$. Then the curve $\tilde{\varphi}(\gamma(t))(0<t \leqq 1)$ in $X$ approaches to the boundary of $X$ as $t \rightarrow 0$, namely, for any compact $C$ in $X$ there is a positive $\delta$ such that $\tilde{\varphi}(\gamma(t)) \notin C$ if $0<t \leqq \delta$. In fact, if a compact $C$ in $X$ contains $\tilde{\varphi}\left(\gamma\left(t_{n}\right)\right)$ for a suitable $\left\{t_{n}\right\}$ with $\lim _{n} t_{n}=0$, we can find easily a sequence $\left\{x_{n}\right\}$ in $X$ with $\lim _{n} x_{n}=x_{0}$ such that $\left\{\varphi_{n}\left(x_{n}\right)\right\}$ has an accumulation point in $X$. Lemma 6. 2 implies $x_{0} \in G$, which is a contradiction.

Now, for each $n$ we define a curve $\gamma_{n}(t)=\varphi_{n}^{-1} \tilde{\varphi}(\gamma(t)) \quad(0<t \leqq 1)$, which approaches to the boundary of $X$ as $t \rightarrow 0$ because $\varphi_{n}$ is a homeomorphism of $X$. By the assumption of Proposition 6. 1, we can take open sets $D^{\prime}, D^{\prime \prime}$ such that $K \subset D^{\prime \prime} \Subset D^{\prime} \Subset D$ and $\varphi(\bar{D}) \subset D$. Then, in virtue of Lemma 3. 2, there exists a positive integer $n_{0}$ such that $\varphi_{m}(K) \subset \varphi\left(D^{\prime \prime}\right) \subset \varphi_{n}\left(D^{\prime}\right) \subset D$ for any $m, n \geqq n_{0}$. For a neighborhood $U^{\prime}:=X-\bar{D}^{\prime}$ of $X-D$, we have $\varphi_{n}^{-1} \varphi_{m}\left(U^{\prime}\right) \subset X-K$ for these $m$ and $n$ and so the mappings $\pi \varphi_{n}^{-1} \varphi_{m}: U^{\prime} \rightarrow C^{N}$ are well-defined. The images of these mappings are included in $\pi(X-K) \Subset C^{N}$. We may apply Theorem 2.1 to the sequence $\left\{\pi \varphi_{n}^{-1} \varphi_{m}\right.$; $\left.m=n_{0}, n_{0}+1, \cdots\right\}$ for each $n \geqq n_{0}$. After a suitable replacement of indices, we may assume each $\left\{\pi \varphi_{n}^{-1} \varphi_{m} ; m=1,2, \cdots\right\}$ converges on $U^{\prime}$ to a mapping $\lambda_{n}$, which is equal to $\pi \varphi_{n}^{-1} \tilde{\varphi}$ on $G$. Moreover, $\left\{\lambda_{n}\right\}$ may be assumed to converge on $U^{\prime}$ to a mapping $\lambda$. Then $\lambda=\lim _{n} \pi \varphi_{n}^{-1} \tilde{\varphi}=\pi$ on $G-D$ and therefore on any connected component of $U^{\prime}$ intersecting $G$. Now, we take relatively compact neighborhoods $V$ of $x_{0}$ and $W$ of $\pi\left(x_{0}\right)$ such that $\pi \mid V: V \rightarrow W$ is proper. For any connected neighborhood $V^{\prime}$ of $x_{0}$ with $V^{\prime} \Subset V, \lambda\left(\bar{V}^{\prime}\right)=\pi\left(\bar{V}^{\prime}\right) \subset W$ implies that

$$
\lambda_{n}\left(V^{\prime} \cap G\right)=\pi \varphi_{n}^{-1} \tilde{\varphi}\left(V^{\prime} \cap G\right) \subset W
$$

for almost all $n$. For a sufficiently small $\delta>0, \quad\{r(t) ; 0<t \leqq \delta\} \subset V^{\prime} \cap G$. So, $\lambda_{n}(\gamma(t))=\pi r_{n}(t) \in W$ for any $t(0<t \leqq \delta)$ and almost all $n$. While, we have

$$
\lim _{n} \gamma_{n}(t)=\lim _{n} \varphi_{n}^{-1} \tilde{\varphi}(\gamma(t))=\gamma(t)
$$

for an arbitrarily fixed $t$ because $\lim _{n} \varphi_{n}^{-1}=\tilde{\varphi}^{-1}$ by Proposition 3. 3. Therefore, $\varphi_{n}^{-1} \tilde{\varphi}\left(V^{\prime} \cap G\right) \cap V \neq \phi$ for a sufficiently large $n$. Since $V$ is open and closed in $\pi^{-1}(W)$, we conclude $\left\{\gamma_{n}(t) ; 0<t \leqq \delta\right\} \subset V$. Hence, $\gamma_{n}(t)$ cannot approach to the boundary of $X$. This is a contradiction. In conclusion, we see $G=X$. 
Proof of Proposition 6. 1. According to Lemma 6. 3 and Lemma 6.4 a given $\left\{\varphi_{n}\right\}$ has a subsequence which converges to a mapping $\tilde{\varphi} \in \operatorname{Hol}(X, X)$. On the other hand, since $\lim _{n} \varphi_{n}=\varphi$ on $D(\neq \phi)$, any convergent subsequence of $\left\{\varphi_{n}\right\}$ has the same limit $\tilde{\varphi}$ in $\operatorname{Hol}(X, X)$ by the theorem of identity. Moreover, any subsequence of $\left\{\varphi_{n}\right\}$ has a convergent subsequence by , the same reason as above. In conclusion, $\left\{\varphi_{n}\right\}$ itself converges to $\tilde{\varphi}$ in $\operatorname{Hol}(X, X)$.

Corollary 6. 5. Let $X$ be a BK-complete complex space and $\left\{\varphi_{n}\right\}$ be a sequence in Aut $(X)$. If for a point $x_{0}\left\{\varphi_{n}\left(x_{0}\right)\right\}$ has an accumlation point in $X$, then $\left\{\varphi_{n}\right\}$ has a convergent subsequence in $\operatorname{Hol}(X, X)$.

Proof. As in the proof of Lemma 6. 3, we consider the set

$$
G=\left\{x ;\left\{\varphi_{n}(x)\right\} \text { has an accumulation point in } X\right\},
$$

which contains $x_{0}$ as an interior point in view of Lemma 6. 2. Moreover, by the same argument as in the proof of Lemma 6. 4, we see $G=X$. And, in view of the proof of Lemma 6. 3, we can easily prove Corollary 6.5.

Proof of Theorem 5. 3. Suppose that $X$ has a compact subset $K$ such that $X-K$ is $B K$-complete, where we may assume $K \neq \phi$. With each $x \in K$ we associate neighborhoods $U_{x}, V_{x}, U_{x}^{\prime}$ and $V_{x}^{\prime}$ of $x$ such that $U_{x} \Subset V_{x} \Subset U_{x}^{\prime} \Subset V_{x}^{\prime} \Subset W$ with some $K$-complete open set $W$. Among these $U_{x}^{\prime} s$, we can select finitely many $U_{x_{i}}(1 \leqq i \leqq r)$ with $K \subset D:=\cup_{i=1}^{r} U_{x_{i}}$. Taking an open set $D^{\prime}$ with $K \subset D^{\prime} \Subset D$, we consider the set

$$
\mathfrak{U}_{1}=U_{i=1}^{r}\left(W\left(\bar{U}_{x_{i}}, V_{x_{i}}\right) \cap W\left(\bar{U}_{x_{i}}^{\prime}, V_{x_{i}}^{\prime}\right)^{-1}\right) \cap W\left(K, D^{\prime}\right),
$$

where for any $\mathfrak{H} \subset \operatorname{Aut}(X) \mathfrak{U}^{-1}$ denotes the set $\left\{\varphi^{-1} ; \varphi \in \mathfrak{U}\right\}$. Obviously, $\mathfrak{u}_{1}$ is a neighborhood of $\varepsilon$ in $\operatorname{Aut}(X)$. We shall prove that the neighborhood $\mathfrak{U}:=\mathfrak{H}_{1} \cap \mathfrak{U}_{1}^{-1}$ of $\varepsilon$ is relatively compact in $\operatorname{Aut}(X)$.

Take an arbitrary sequence $\left\{\varphi_{n}\right\}$ in $\mathfrak{u}$. For each $i, \varphi_{n}\left(U_{x_{i}}\right) \subset V_{x_{i}}$. According to Theorem 2. 1, each $\left\{\varphi_{n} \mid U_{x_{i}}\right\}$ has a convergent subsequence. Choosing subsequences repeatedly, we obtain a subsequence $\left\{\varphi_{n_{k}}\right\}$ of $\left\{\varphi_{n}\right\}$ which converges to a mapping $\varphi$ on $D$. By the definition of $\mathfrak{U}$, we see $\varphi(K) \subset \bar{D}^{\prime} \subset D$ and $\varphi(D) \subset \cup_{i=1}^{r} \bar{V}_{x_{i}} \subset D^{\prime \prime}:=\cup_{i=1}^{r} U_{x_{i}}^{\prime}$. By the same argument, after a suitable replacement of indices, we may assume that for the above 
$n_{k}^{\prime} s\left\{\varphi_{n_{k}}^{-1}\right\}$ converges also to a mapping $\psi$ on $D^{\prime \prime}$, which satisfies $\psi(K) \subset \bar{D}^{\prime} \subset D$. The mappings $\varphi \psi$ and $\psi \varphi$ are well-defined and equal to $\varepsilon$ on $K$ and on $D$ respectively. Therefore, $\varphi$ is injective on $D$ and $\psi(K) \subset D$ means $K \subset \varphi(D)$. The sequence $\left\{\varphi_{n_{k}}\right\}$ satisfies all conditions in Proposition 6.1 for the above $D$. So, the sequence $\left\{\varphi_{n}\right\}$ has a convergent subsequence $\left\{\varphi_{n_{k}}\right\}$ with the limit $\tilde{\varphi}$ in $\mathrm{Hol}(X, X)$. On the other hand, $\mathfrak{u}$ is symmetry. We can apply the same argument to the sequence $\left\{\varphi_{n}^{-1}\right\}$. Thus, by the usual method, we conclude $\tilde{\varphi} \in \operatorname{Aut}(X)$. This shows that $\mathfrak{U}$ is relatively compact and hence $\operatorname{Aut}(X)$ is locally compact.

\section{\$7. A convergence theorem of injective holomorphic mappings.}

In the previous paper [8], we introduce the notion of $*$-strongly $s$ convexity. By definition, a real-valued function $v$ on $X$ is *-strongly $s$ convex at $x \in X$ if and only if there exist a nowhere degenerate holomorphic mapping $\pi$ of a neighborhood $V$ of $x$ into a domain $D \subset C^{M}$ and a strongly $s$-convex function $\tilde{v}$ on $D$ such that $v=\tilde{v} \pi$ on $V$ ([8], Definition 2. 5, p. 55). An open set $D$ in $X$ is called to be $*$-strongly $s$-concave at $x \in X$ if it holds $D \cap V=\{v>v(x)\}$ with a suitable $v$ on a neighborhood $V$ of $x$ which is *-strongly $s$-convex at $x$ ([8], Definition 2. 8, p. 56).

For example, we see

(7. 1) Let $M$ be an analytic subset of a complex space $X$. If $\operatorname{dim}_{x} M=s-1$, $X-M$ is *-strongly s-concave at $x$.

Indeed, for a suitable neighborhood $U$ of $x$ in $X, M$ is represented as

$$
M \cap U=\left\{f_{1}=\cdots=f_{k}=0\right\}
$$

with holomorphic functions $f_{i}(1 \leqq i \leqq k)$ in $U$. We define the function $v=\left|f_{1}\right|^{2}+\cdots+\left|f_{k}\right|^{2}$ on $U$, which satisfies $\{v>0\} \cap U=U-(M \cap U)$. Considering the mapping $\pi=\left(f_{1}, \cdots, f_{k}\right)$ of $U$ into $C^{k}$ and $\tilde{v}:=\left|z_{1}\right|^{2}+\cdots$ $+\left|z_{k}\right|^{2}$ on $C^{k}$, we see easily that $v=\tilde{v} \pi, \operatorname{dim}_{x} \pi^{-1} \pi(x)=\operatorname{dim}_{x} M=s-1$ and $\tilde{v}$ is strongly 1-convex at $\pi(x)$. In view of Example 2. 6, (iii) in [8], p. 55, $v$ is $*$-strongly $s$-convex at $x$.

Lemma 7. 2. Let $D$ be an open set in a normal $X$ which is *-strongly $(N-1)$-concave at $x \in X(N=\operatorname{dim} X)$. Then there exists a connected neighborhood $U$ of $x$ such that any $(N-1)$-dimensional analytic subset of $U$ intersects $D$ and, 
moreover, for an open set $G$ with $G \Subset D$ and $G \subset U$, every holomorphic function on $G$ is uniquely continuable to $U$.

For the proof, see [8], Corollary 5. 4, p. 69 and Proposition 4. 3, p. 64. In view of the proof of these assertions, we can conclude easily Lemma 7.2 with a little modification.

Lemma 7. 3. Assume that for normal complex spaces $X$ and $Y$, a sequence $\left\{\varphi_{n}\right\}$ converges to $\varphi$ in $\operatorname{Hol}(X, Y)$. If each $\varphi_{n}$ is injective and $E=\left\{x ; \operatorname{dim}_{x} \varphi^{-1} \varphi(x)>0\right\}$ is of codimension $\geqq 2$, then $\varphi$ is also injective.

Proof. Under the assumption $\operatorname{codim} E \geqq 2$, take two distinct points $x_{1}, x_{2}$ in $X$ and Stein neighborhoods $U_{1}$ of $x_{1}$ and $U_{2}$ of $x_{2}$ such that $\bar{U}_{1} \cap \bar{U}_{2}=\phi . \quad$ In view of (7. 1$), X-E$ is $*$-strongly $(N-1)$-concave at any $x \in E(N=\operatorname{dim} X)$. By Lemma 7.2, for each $i=1$, 2, there exists an open set $G_{i}$ such that $G_{i} \Subset U_{i}, G_{i} \cap E=\phi$ and every holomorphic function on $G_{i}$ is continuable to a neighborhood $V_{i}$ of $x_{i}$. Taking an arbitrary $G_{i}^{\prime}$ with $G_{i} \subset G_{i}^{\prime} \Subset U_{i}-E(i=1,2)$, we see $\varphi_{m}\left(G_{i}\right) \subset \varphi\left(G_{i}^{\prime}\right) \subset \varphi_{n}\left(U_{i}\right)$ for almost all $m$ and $n$ by Lemma 3.2. For a sufficiently large $n_{0}, \varphi_{n}\left(G_{i}\right)\left(n \geqq n_{0}\right)$ are included in a Stein space $\varphi_{n_{0}}\left(U_{i}\right)$. By H. Kerner's theorem ([15] and [8]), $\varphi_{n} \mid G_{i}$ is continuable to a mapping $\tilde{\varphi}_{n}: V_{i} \rightarrow \varphi_{n_{0}}\left(U_{i}\right)$, which is the original $\varphi_{n}$ on $V_{i}$. This means $\varphi_{n}\left(V_{i}\right) \subset \varphi_{n_{0}}\left(U_{i}\right)$ and particularly $\varphi_{n}\left(x_{i}\right) \in \varphi_{n_{0}}\left(U_{i}\right)$. As $n \rightarrow \infty$, we see $\varphi\left(x_{i}\right) \in \overline{\varphi_{n_{0}}\left(U_{i}\right)} \quad(i=1,2)$. While, $\bar{U}_{1} \cap \bar{U}_{2}=\phi$ implies $\overline{\varphi_{n_{0}}\left(U_{1}\right)} \cap \overline{\varphi_{n_{0}}\left(U_{2}\right)}=\phi$ whence $\varphi\left(x_{1}\right) \neq \varphi\left(x_{2}\right)$. This shows that $\varphi$ is injective on $X$.

REMARK. In Lemma 7.2, if $X$ is a complex manifold, we can assert $E=\phi$ or $E=X$ without the assumption codim $E \geqq 2$, considering the jacobians of mappings (C. Carathéodory [5], Satz 6, p. 719).

After these preparations, we can prove

Proposition 7. 4. Let $D$ be an open set in a normal complex space $X$ which is *-strongly $(N-1)$-concave at $x \in X(N=\operatorname{dim} X)$ and $\left\{\varphi_{n}\right\}$ be a sequence of injective holomorphic mappings of $X$ into another normal $Y$. If $\left\{\varphi_{n}\right\}$ converges on $D$ to a nowhere degenerate $\varphi \in \operatorname{Hol}(D, Y),\left\{\varphi_{n}\right\}$ converges also on a neighborhood $U$ of $x$ to an injective mapping $\tilde{\varphi}$.

Proof. Take a Stein neighborhood $V$ of $x$ which is relatively compact in some $K$-complete open subset of $X$. By Lemma 7.2 there exists a con- 
nected neighborhood $U$ of $x(U \subset V)$ such that any $(N-1)$-dimensional analytic subset of $U$ intersects $D$ and every holomorphic function on $G$ is uniquely continuable to $U$ for some $G \Subset V \cap D$. As in the proof of Lemma 7. 3, taking an arbitrary $G^{\prime}$ with $G \Subset G^{\prime} \Subset D \cap V$, we see $\varphi_{n}(G) \subset \varphi\left(G^{\prime}\right) \subset \varphi_{n}(V)$ for any $n \geqq n_{0}$ with a suitable $n_{0}$ by Lemma 3.2. While, since $\varphi_{n}$ maps $X$ biholomorphically onto an open subset of $Y, \varphi_{n_{0}}(V)$ is Stein and relatively compact in some $K$-complete set. As in the proof of Lemma 7. 3, we see $\varphi_{n}(U) \subset \varphi_{n_{0}}(V)$. Then, we can select a subsequence of $\left\{\varphi_{n}\right\}$ which converges on $U$ by Theorem 2. 1. Since $\lim _{n} \varphi_{n}=\varphi$ on $D \cap U$, any convergent subsequence of $\left\{\varphi_{n}\right\}$ has the same limit $\tilde{\varphi}$ on $U$ by the theorem of identity. This concludes $\lim _{n} \varphi_{n}=\tilde{\varphi}$ in $\operatorname{Hol}(U, Y)$. To see the injectivity of $\tilde{\varphi}$, we consider the set $E=\left\{x \in U\right.$; $\left.\operatorname{dim}_{x} \tilde{\varphi}^{-1} \tilde{\varphi}(x)>0\right\}$, which is analytic in $U$. By the assumption, $E \cap D=\phi$ and so $\operatorname{codim} E \geqq 2$ because of the property of $U$. Then $\tilde{\varphi}$ is injective in virtue of Lemma 7.3.

We have also the following convergence theorem of injective holomorphic mappings.

THeORem 7.5. Let $X$ and $Y$ be connected normal complex spaces of dimension $N$ and $v$ be $a$ *strongly $(N-1)$-convex function on $X$ with $v>c_{0}(\geqq-\infty)$ such that

$$
\left\{x \in X ; c<v(x) \leqq c_{1}\right\} \Subset X
$$

if $c_{0}<c<c_{1}$ for a fixed $c_{1}$. If a sequence $\left\{\varphi_{n}\right\}$ of injective mappings in $\operatorname{Hol}(X, Y)$ converges to a nowhere degenerate mapping on the set $\left\{x \in X ; v(x)>c_{1}\right\}$, then $\left\{\varphi_{n}\right\}$ converges on the total $X$ to an injective $\varphi \in \operatorname{Hol}(X, Y)$.

Proof. As usual, we consider the set $\Gamma$ of all real numbers $c$ such that $\left\{\varphi_{n}\right\}$ converges on $X_{c}:=\{x \in X ; v(x)>c\}$ to an injective $\varphi_{c} \in \operatorname{Hol}\left(X_{c}, Y\right)$. Our aim is to show $\min \Gamma=c_{0}$. Assume $c_{2}:=\inf \Gamma=\min \Gamma>c_{0}$. By Theorem 7. 4, each $x \in L:=\left\{v=c_{2}\right\}$ has a neighborhood $U_{x}$ such that $\left\{\varphi_{n}\right\}$ converges to an injective $\tilde{\varphi} \in \operatorname{Hol}\left(U_{x}, Y\right)$ on $U_{x}$. Covering $L$ by finitely many such $U_{x_{i}}(1 \leqq i \leqq r)$, we get an open set $G:=\cup_{i=1}^{r} U_{x_{i}} \supset L$ such that $\left\{\varphi_{n}\right\}$ converges on $G$ to an injective mapping. Then, $c_{3}:=\sup \{v(x)$; $\left.x \notin G \cup X_{c_{2}}\right\}<c_{2}$ and $c_{3} \in \Gamma$. This is a contradiction. We conclude $\left\{\varphi_{n}\right\}$ converges on $X=X_{c_{0}}$ to an injective $\varphi \in \operatorname{Hol}(X, Y)$. 


\section{§8. The automorphism group of a $*$-strongly pseudo-concave com- plex space.}

Firstly, we give

Definition 8. 1. We shall call a purely $N$-dimensional complex space $X$ to be $*$-strongly pseudo-concave if there exists a continuous function $v$ with $v>c_{0}(\geqq-\infty)$ on $X$ such that $v$ is $*$-strongly $(N-1)$-convex at any point in $X-K$ for a compact subset $K$ of $X$ and satisfies the condition

$$
\{x \in X ; v(x)>c\} \Subset X
$$

for any $c>c_{0}$ (c.f. Andreotti-Grauert [1], p. 236).

An important example of a *-strongly pseudo-concave space is given as follows.

ExAmple 8. 2. Let $X$ be an $N$-dimensional connected compact complex manifold and $M$ be an analytic set in $X$. If $M$ is of embedding dimension $\leqq N-2$, namely, $\operatorname{dim}_{C} \mathfrak{m}_{x / \mathfrak{m}_{x}^{2}} \leqq N-1$ for the maximal ideal $\mathfrak{m}_{x}$ of the local ring of all germs of holomorphic functions on $M$ at each $x \in M$, then $X-M$ is *-strongly pseudo-concave.

Indeed, for each $x \in M M$ can be represented as

$$
M \cap U=\left\{f_{1}=\cdots=f_{k}=0\right\}
$$

with holomorphic functions $f_{i}(1 \leqq i \leqq k)$ on a neighborhood $U$ of $x$. By the assumption, we may assume that they satisfy

$$
\operatorname{rank} \frac{\partial\left(f_{1}, \cdots, f_{k}\right)}{\partial\left(u_{1}, \cdots, u_{N}\right)} \geqq 2
$$

on $U$, where $\left\{u_{1}, \cdots, u_{N}\right\}$ is a system of local coordinates in $U$. As is easily seen, the function $v_{x}=\left|f_{1}\right|^{2}+\cdots+\left|f_{k}\right|^{2}$ on $U$ is strongly $(N-1)$ convex at $x$ and satisfies $U-(M \cap U)=\left\{v_{x}>0\right\}$. In this situation, by the same argument as in the proof of Proposition 15 in [1], p. 234, we can construct a strongly $(N-1)$-convex function $v$ on a neighborhood $D$ of $M$ such that $D-M=\{v>0\}$, because $M$ is compact. Let $c=\min \{v(x)$; $\left.x \in \partial D^{\prime}\right\}(>0)$ for some $D^{\prime}$ with $M \subset D^{\prime} \Subset D$. Putting $v^{\prime}=c$ on $K:=\left(X-D^{\prime}\right)$ $\cup\{v \geqq c\}$ and $=v$ on $\{v<c\} \cap D^{\prime}$, we see easily the function $v^{\prime}$ satisfies the conditions in Definition 8. 1 for the space $X-M$. 
Now, we give the following main theorem.

Theorem 8. 3. Let $X$ be an irreducible *-strongly pseudo-concave complex space. Then $\operatorname{Aut}(X)$ is locally compact and so a Lie group.

Proof. We have only to prove the local compactness of Aut $(X)$ as stated in \$5. Moreover, $X$ may be assumed to be normal in virtue of Proposition 4.2 and connected by the assumption. Let $v$ be a continuous function on $X$ with the property in Definition 8.1. We take a real $c_{1}$ such that $c_{0}<c_{1}<\inf \{v(x) ; x \in K\}$. The set $L=\left\{x ; v(x) \geqq c_{1}\right\}$ is compact and includes $K$. With each $x \in L$ we associate neighborhoods $U_{x}, V_{x}, U_{x}^{\prime}$ and $V_{x}^{\prime}$ of $x$ such that $U_{x} \Subset V_{x} \Subset U_{x}^{\prime} \Subset V_{x}^{\prime} \Subset W$ with some $K$-complete open set $W$. Covering $L$ by finitely many $U_{x_{i}}(1 \leqq i \leqq r)$ and putting $D:=\cup_{i=1}^{r} U_{x_{i}}$, we consider the set

$$
\mathfrak{U}=\cap_{i=1}^{r}\left(W\left(\bar{U}_{x_{i}}, V_{x_{i}}\right) \cap W\left(\bar{U}_{x_{i}}^{\prime}, V_{x_{i}}^{\prime}\right)^{-1}\right) \cap W(L, D)^{-1},
$$

which is a neighborhood of $\varepsilon$ in $\operatorname{Aut}(X)$. We shall prove that $\mathfrak{u}$ is relatively compact in Aut $(X)$. As in the proof of Theorem 5. 3, any sequence $\left\{\varphi_{n}\right\}$ in $\mathfrak{U}$ has a subsequence $\left\{\varphi_{n_{k}}\right\}$ which converges on $D$ to a mapping $\varphi \in \operatorname{Hol}(D, X)$. Moreover, we may assume that $\left\{\varphi_{n_{k}}^{-1}\right\}$ converges also on $D^{\prime}:=\cup_{i} U_{x_{i}}^{\prime}$ to $\psi \in \operatorname{Hol}\left(D^{\prime}, X\right)$. By the definition of $\mathfrak{H}, \varphi(D) \subset \cup_{i} \bar{V}_{x_{i}} \subset D^{\prime}$. The composite $\psi \cdot \varphi$ is well-defined and equal to $\varepsilon$ on $D$, whence $\varphi$ is injective on $D$. Moreover, since $D \supset X_{c_{1}}:=\left\{x ; v(x)>c_{1}\right\}$, the mappings $\varphi_{n_{k}} \in \operatorname{Aut}(X) \subset \operatorname{Hol}(X, X)$ satisfy all assumptions in Theorem 7. 5. The sequence $\left\{\varphi_{n_{k}}\right\}$ converges to a mapping $\tilde{\varphi} \in \operatorname{Hol}(X, X)$. While, $\lim _{k} \varphi_{n_{k}}^{-1}=\psi$ is injective on $\varphi(D)$. Since $\phi(D) \supset X_{c}$ with a suitable $c$, we can apply the same argument to the mappings $\varphi_{n_{k}}^{-1}$. We obtain the limit $\tilde{\phi} \in \operatorname{Hol}(X, X)$ of $\varphi_{t_{k}}^{-1}$, which satisfies $\phi \tilde{\psi}=\tilde{\phi} \psi=\varepsilon$ on $X$. Therefore, $\tilde{\phi} \in \operatorname{Aut}(X)$ and so $\mathfrak{U}$ is relatively compact.

According to Example 8. 2, we have

Corollary 8. 4. Let $X$ be an $N$-dimensional connected compact complex manifold and $M$ be an analytic set of embedding dimension $\leqq N-2$ in $X$. Then Aut $(X)$ has a structure of a Lie group.

Remark. If emdim $M=N-1$, Corollary 8.4 is false. Let $P^{N}$ be the 
$N$-dimensional projective space with homogeneous coordinates $z_{0}, z_{1}, \cdots, z_{N}$. The space $P^{N}-\left\{z_{0}=0\right\}$ is biholomorphically isomorphic with $C^{N}$. As is well known, $\operatorname{Aut}\left(C^{N}\right)$ is not locally compact with the compact open topology if $N \geqq 2$.

\section{REFERENCES}

[1] A. Andreotti and $\mathrm{H}$. Grauert, Théorèmes de finitude pour la cohomologie des espaces complexes, Bull. Soc. Math. France, 90 (1962), 193-259.

[2] S. Bochner and D. Montgomery, Locally compact groups of differentiable transformations, Ann. Math., 47 (1946), 639-653.

[3] S. Bochner and D. Montgomery, Groups on analytic manifolds, Ann. Math., 48 (1947), 659-669.

[4] N. Bourbaki, Fasc. X, Topologie générale, Chap. 10, Hermann, Paris, 1961.

[5] C. Carathéodory, Über die Abbildungen, die durch Systeme von analytischen Funktionen von mehreren Veränderlichen erzeugt werden, Math. Z., 34 (1931), 758-792.

[6] H. Cartan, Sur les fonctions de plusieurs variables complexes. L'itération des transformations intérieures d'un domaine borné, Math. Z., 35 (1932), 760-773.

[ 7 ] H. Cartan, Sur les groupes de transformations analytiques, Act. Sci. Ind., 198, Paris, 1935.

[ 8 ] H. Fujimoto, On the continuation of analytic sets, J. Math. Soc. Japan, 18 (1966), 51-85.

[9] H. Grauert, Charakterisierung der holomorph-vollständigen komplexer Räume, Math. Ann., 129 (1955), 233-259.

[10] H. Grauert and R. Remmert, Komplexe Räume, Math. Ann., 136 (1958) 245-318.

[11] R.C. Gunning, On Vitali's theorem for complex spaces with singularities, J. Math. Mech., 8 (1959), 133-141.

[12] T. Husain, Introduction to topological groups, W.B. Saunders Company, Philadelphia and London, 1966.

[13] K. Kasahara, On Hartogs-Osgood's theorem for Stein spaces, J. Math. Soc. Japan, 17 (1965), 297-312.

[14] W. Kaup, Reelle Transformationsgruppen und invariante Metriken auf komplexen Räumen, Inv. math., 3 (1967), 43-70.

[15] H. Kerner, Über die Fortsetzung holomorpher Abbildungen, Arch. Math., 11 (1960), 44-49.

[16] H. Kerner, Über die Automorphismengruppen kompakter komplexer Räume, Arch. Math., 11 (1960), 282-288.

[17] M. Kuranishi, On conditions of differentiability of locally compact groups, Nagoya Math. J., 1 (1950) 71-81.

[18] D. Montgomery and L. Zippin, Topological transformation groups, Interscience Publ., Inc., New York, 1955.

[19] R. Remmert, Holomorphe und meromorphe Abbildungen komplexer Räume, Math. Ann., 133 (1957), 328-370. 